\title{
ERGODIC PROPERTIES AND ERGODIC DECOMPOSITIONS OF CONTINUOUS-TIME MARKOV PROCESSES
}

\author{
O. L. V. COSTA, ${ }^{*}$ Escola Politécnica da Universidade de São Paulo \\ F. DUFOUR, ${ }^{* *}$ Université Bordeaux I
}

\begin{abstract}
In this paper we obtain some ergodic properties and ergodic decompositions of a continuous-time, Borel right Markov process taking values in a locally compact and separable metric space. Initially, we assume that an invariant probability measure (IPM) $\mu$ exists for the process and, without making any further assumptions on the transition kernel, obtain some characterization results for the convergence of the expected occupation measure to a limit kernel. Under the same assumption, we present the so-called Yosida decomposition. Next, instead of assuming the existence of an IPM, we assume that the Markov process satisfies a certain condition, named the $T^{\prime}$-condition. Under this condition it is shown that the Foster-Lyapunov criterion is necessary and sufficient for the existence of an IPM and that the process admits a Doeblin decomposition. Furthermore, it is shown that in this case the set of ergodic probability measures is countable and that every probability measure for the Markov process is nonsingular with respect to the transition kernel.
\end{abstract}

Keywords: Markov process; continuous time; invariant probability measure; limit kernel; Foster-Lyapunov criterion; ergodic decomposition; Doeblin decomposition; Yosida decomposition

2000 Mathematics Subject Classification: Primary 60J10

\section{Introduction}

In recent years a great deal of attention has been given to ergodic properties of Markov processes. For the discrete-time case, we refer to Hernández-Lerma and Lasserre (1998) and Meyn and Tweedie (1993a) for rather complete discussions on this subject. In general the analysis of the continuous-time case is made by looking at the ergodic properties of some associated discrete-time Markov chains. This approach was taken in, for instance, Tuominen and Tweedie (1979), Meyn and Tweedie (1993b), and Meyn and Tweedie (1993c), which comprise only a small sample of papers on this subject. In this paper we derive some ergodic properties and ergodic decompositions for a continuous-time, Borel right Markov process taking values in a locally compact and separable metric space. As in Tuominen and Tweedie (1979), we analyse the continuous-time case by looking at the ergodic properties of some associated discrete-time Markov chains.

Initially, we assume that an invariant probability measure (IPM) $\mu$ exists for the process and, without making any further assumptions on the transition kernel, obtain some characterization

Received 11 October 2005; revision received 23 March 2006.

* Postal address: Departamento de Engenharia de Telecomunicações e Controle, Escola Politécnica da Universidade de São Paulo, CEP 05508 900, São Paulo, Brazil. Email address: oswaldo@lac.usp.br

** Postal address: Mathématiques Appliqées de Bordeaux, Université Bordeaux I, 351 cours de la Liberation, 33405

Talence Cedex, France. Email address: dufour@math.u-bordeaux1.fr 
results for the convergence of the expected occupation measure to a limit kernel. We follow an approach closely related to that of Hernández-Lerma and Lasserre (1998). It is shown that this limit kernel can be written $\mu$-almost everywhere ( $\mu$-a.e.) as a composition of the limit kernel associated with an embedded discrete-time Markov chain of the location of the process at time 1 with an expected value operator of the integral of the process up to time 1 . Moreover, weak convergence of the expected occupation measure to the limit kernel occurs $\mu$-a.e. In relation to the ergodic decomposition of a continuous-time Markov process, we present the socalled Yosida decomposition, which is the counterpart of the results obtained by Yosida (1980, pp. 393-397) and Hernández-Lerma and Lasserre (1998) in the discrete-time context. The Yosida decomposition shows that if an ergodic measure for the continuous-time process exists, then there also exists an indecomposable maximal closed set in which the ergodic measure is the unique IPM for the continuous-time process (see Proposition 5.2, below).

We then replace the assumption of the existence of an IPM by the assumption that the Markov process satisfies a certain condition, named the $T^{\prime}$-condition. As will be pointed out in some examples presented in Section 6, $T$-processes and irreducible processes in continuous time are Markov processes satisfying the $T^{\prime}$-condition, but the converse is not in general true. In this sense, processes satisfying the $T^{\prime}$-condition can be seen as abstract generalizations of $T$-processes and irreducible processes. It is shown that the so-called Doeblin decomposition for the state space of the Markov process holds for a process satisfying the $T^{\prime}$-condition. Our result is related to the Doeblin decomposition studied by Tuominen and Tweedie (1979), Tweedie (1979), and Meyn and Tweedie (1993d), (1993b). Under the $T^{\prime}$-condition, it is shown that the Foster-Lyapunov criterion is necessary and sufficient to ensure the existence of an IPM. To the best of the authors' knowledge, this result seems to be the most general attempt to show that the Foster-Lyapunov criterion is a necessary condition to ensure the existence of such a measure. The results of this part of the paper are related to the results obtained by the authors in the discrete-time context (see Costa and Dufour (2005b)). Moreover, the Foster-Lyapunov criterion is shown to ensure both the decomposition of the set of ergodic IPMs into a countable set of IPMs nonsingular with respect to the transition kernel (see Definition 6.5, below) and that every ergodic IPM is nonsingular with respect to the transition kernel.

The paper is organized as follows. In Section 2 we recall some classical definitions related to Markov processes. Our notation is similar to that of Tuominen and Tweedie (1979). In Section 3 we recall some limiting results for the occupation measure for a Markov chain, and some recurrence structures relating continuous- and discrete-time Markov processes. In Section 4 we present some characterization results for the convergence of the expected occupation measure of the continuous-time process to a limit kernel, and in Section 5 deal with the so-called Yosida decomposition, under the assumption that an IPM exists for the continuous-time process. The results related to the Doeblin decomposition and the Foster-Lyapunov criterion are presented in Section 6, under the assumption that the continuous-time process satisfies the $T^{\prime}$-condition. It is also shown in this section that the set of ergodic measures is countable and, furthermore, that each ergodic IPM is nonsingular with respect to the transition kernel.

\section{Definitions}

Denote the set of nonnegative reals by $\mathbb{R}_{+}$, and let $\mathbb{R}_{+}^{*}:=\mathbb{R}_{+} \backslash\{0\}$ and $\mathbb{N}^{*}:=\mathbb{N} \backslash\{0\}$. Let $X$ be a locally compact and separable metric space and $\mathscr{B}$ the Borel field on $X$. For any probability measure $\mu$ on $X$, we let $L_{1}(\mu):=L_{1}(X, \mathcal{B}, \mu)$, the space of real-valued, measureable functions $f$ on $(X, \mathcal{B})$ such that $\mu(|f|):=\int_{X}|f(x)| \mu(\mathrm{d} x)<\infty$. For any $A \in \mathcal{B}, A^{\mathrm{c}}:=X \backslash A$ and $\mathbf{1}_{A}(x)$ is the indicator function associated with $A$. For two measures $\mu$ and $v$ defined on the same 
measurable space, we write $\mu \ll v$ to mean that $\mu$ is absolutely continuous with respect to $v$. In this paper we consider two types of time-homogeneous Markov process: the discrete-time Markov chain $\left\{\Psi_{n}\right\}_{n \in \mathbb{N}}$, where the associated transition probability function is denoted by $G$, and the continuous-time Markov process $\left\{X_{t}\right\}_{t \in \mathbb{R}_{+}}$. We shall assume that $\left\{X_{t}\right\}_{t \in \mathbb{R}_{+}}$is a Borel right process (see Sharpe (1988, pp. 104-105)) where the associated transition semigroup is denoted by $\left\{P^{t}\right\}_{t \in \mathbb{R}_{+}}$, and for every $t \in \mathbb{R}_{+}$shall write $P^{t}(x, A)=\mathrm{P}_{x}\left(X_{t} \in A\right)$, where $X_{0}=x \in X$ is the initial condition and $A \in \mathscr{B}$.

We recall now some classical definitions related to Markov processes. For a complete exposition on the subject, the reader is referred to Meyn and Tweedie (1992), (1993a), (1993b), (1993c). Let us introduce the first hitting time of the set $A \in \mathscr{B}$ and the number of visits to the set $A$, respectively $\tau_{A}^{\Psi}:=\inf \left\{n \geq 1: \Psi_{n} \in A\right\}$ and $\eta_{A}^{\Psi}:=\sum_{n=0}^{\infty} \mathbf{1}_{A}\left(\Psi_{n}\right)$ in the discrete-time case, and $\tau_{A}^{X}:=\inf \left\{t \in \mathbb{R}^{+}: X_{t} \in A\right\}$ and $\eta_{A}^{X}:=\int_{0}^{\infty} \mathbf{1}_{A}\left(X_{t}\right) \mathrm{d} t$ in the continuous-time case. We also define

$$
L^{\Psi}(x, A):=\mathrm{P}_{x}\left(\tau_{A}^{\Psi}<\infty\right), \quad L^{X}(x, A):=\mathrm{P}_{x}\left(\tau_{A}^{X}<\infty\right),
$$

for all $(x, A) \in X \times \mathcal{B}$.

Some of the following definitions will be presented only for the continuous-time process $\left\{X_{t}\right\}_{t \in \mathbb{R}_{+}}$, but also hold for the discrete-time chain $\left\{\Psi_{k}\right\}_{k \in \mathbb{N}}$ with the superscript ' $X$ ' replaced by ' $\Psi$ '.

Definition 2.1. A set $A \in \mathscr{B}$ is said to be uniformly transient if there exists a constant, $M$, such that $\mathrm{E}_{x}\left(\eta_{A}^{X}\right) \leq M$ for all $x \in A$. A set $B \in \mathscr{B}$ is said to be transient if it has a countable cover consisting of uniformly transient sets. A set $E \in \mathscr{B}$ is said to be absorbing for $\left\{X_{t}\right\}_{t \in \mathbb{R}_{+}}$or for $\left\{\Psi_{n}\right\}_{n \in \mathbb{N}}$ if $E \neq \varnothing$ and if $P^{t}(x, E)=1$ for all $x \in E$ and $t \in \mathbb{R}_{+}$or, respectively, $G(x, E)=1$ for all $x \in E$. A set $E \in \mathscr{B}$ is called closed for $\left\{X_{t}\right\}_{t \in \mathbb{R}_{+}}$or for $\left\{\Psi_{n}\right\}_{n \in \mathbb{N}}$ if $E \neq \varnothing$ and if, for all $x \in E, \mathrm{P}_{x}\left(X_{t} \in E\right.$ for all $\left.t \in \mathbb{R}_{+}\right)=1$ or, respectively, $\mathrm{P}_{x}\left(\Psi_{n} \in E\right.$ for all $\left.n \in \mathbb{N}\right)=1$.

In what follows, for any $A \in \mathscr{B}$ we define $\mathcal{M}^{X}(A) \in \mathscr{B}$ by

$$
\mathcal{M}^{X}(A):=\left\{x \in X: \mathrm{P}_{x}\left(\eta_{A}^{X}=\infty\right)=1\right\} .
$$

Definition 2.2. A set $H \in \mathscr{B}$ is called a Harris set for $\left\{X_{t}\right\}_{t \in \mathbb{R}_{+}}$if it is closed and if there exists a $\sigma$-finite measure $\varphi(\cdot)$ on $\mathscr{B}$ such that, for $\varphi(A)>0, \mathrm{P}_{x}\left(\eta_{A}^{X}=\infty\right)=1$ for all $x \in H$. A closed set $A \in \mathscr{B}$ is said to be maximal closed if $A=\mathcal{M}^{X}(A)$, that is, if $x \in A \Leftrightarrow \mathrm{P}_{x}\left(\eta_{A}^{X}=\infty\right)=1$. We say that $H \in \mathscr{B}$ is a maximal Harris set if it is both Harris and maximal closed.

Definition 2.3. If $b=\left\{b_{k}\right\}_{k=0}^{\infty}$ is a probability on $\mathbb{N}$, then the stochastic kernel $K_{b}^{\Psi}$ associated with the discrete-time Markov chain $\left\{\Psi_{k}\right\}_{k \in \mathbb{N}}$ is defined on $X \times \mathscr{B}$ by

$$
K_{b}^{\Psi}(x, A):=\sum_{k=0}^{\infty} b_{k} G^{k}(x, A) \quad \text { for all } x \in X \text { and all } A \in \mathscr{B} .
$$

If $F$ is a probability distribution on $\mathbb{R}_{+}$, then the stochastic kernel $K_{F}^{X}$ associated with the continuous-time Markov process $\left\{X_{t}\right\}_{t \in \mathbb{R}_{+}}$is defined on $X \times \mathscr{B}$ by

$$
K_{F}^{X}(x, A):=\int_{0}^{\infty} P^{t}(x, A) F(\mathrm{~d} t) \quad \text { for all } x \in X \text { and all } A \in \mathcal{B} .
$$

The resolvent associated with the transition semigroup $\left\{P^{t}\right\}_{t \in \mathbb{R}_{+}}$is denoted by

$$
R(x, A):=\int_{0}^{\infty} P^{t}(x, A) \mathrm{e}^{-t} \mathrm{~d} t .
$$


Definition 2.4. A set $C \in \mathscr{B}$ is called a petite set for $\left\{X_{t}\right\}_{t \in \mathbb{R}_{+}}$or for $\left\{\Psi_{n}\right\}_{n \in \mathbb{N}}$ if there exist both a probability distribution $F$ on $\mathbb{R}_{+}^{*}$ or, respectively, a probability $b$ on $\mathbb{N}^{*}$ and a nontrivial measure $v$ on $(X, B)$ such that, for all $A \in \mathscr{B}$ and all $x \in C, K_{F}^{X}(x, A) \geq v(A)$ or, respectively, $K_{b}^{\Psi}(x, A) \geq v(A)$.

The extended generator $\mathcal{A}$ for $\left\{X_{t}\right\}_{t \in \mathbb{R}_{+}}$and its domain, $D(\mathcal{A})$, are defined next (see also Down et al. (1995, pp. 1675-1676)).

Definition 2.5. Define $D(\mathcal{A})$ as the set of all measurable functions $V: X \times \mathbb{R}_{+} \rightarrow \mathbb{R}$ for which there exists a measurable function $U: X \times \mathbb{R}_{+} \rightarrow \mathbb{R}$ such that, for each $x \in X$ and $t>0$,

$$
\begin{gathered}
\mathrm{E}_{x}\left(\left|\int_{0}^{t} U\left(X_{s}, s\right) \mathrm{d} s\right|\right)<\infty, \\
\mathrm{E}_{x}\left(V\left(X_{t}, t\right)\right)=V(x, 0)+\mathrm{E}_{x}\left(\int_{0}^{t} U\left(X_{s}, s\right) \mathrm{d} s\right) .
\end{gathered}
$$

The extended generator $\mathcal{A}$ for $\left\{X_{t}\right\}_{t \in \mathbb{R}_{+}}$is defined for $V \in D(\mathcal{A})$ by $\mathcal{A} V:=U$, and $D(\mathcal{A})$ is referred to as the domain of $\mathcal{A}$.

We conclude this section by recalling the so-called Doeblin decomposition (since $\mathcal{B}$ is assumed to be countably generated; see Tuominen and Tweedie (1979)).

Definition 2.6. The process $\left\{X_{t}: t \in \mathbb{T}\right\}$ has a Doeblin decomposition if the space $X$ can be expressed as $X=E \cup \bigcup_{n \in \mathcal{I}} H_{n}$, where $\mathcal{I}$ is countable, $H_{n}$ is a sequence of maximal disjoint Harris sets, and $E$ is a transient set.

\section{Occupation measure and recurrence structure of Markov processes}

In this section we recall some limiting results about the occupation measure for a Markov chain, and some recurrence structures relating continuous- and discrete-time Markov processes. Let $G$ be a stochastic kernel associated with a discrete-time Markov chain, and suppose that $v$ is an IPM for $G$. The $n$-step expected occupation measure $G^{(n)}$ associated with the kernel $G$ is defined, for any $x \in X$ and $B \in \mathcal{B}$, as

$$
G^{(n)}(x, B):=\frac{1}{n} \sum_{k=0}^{n-1} G^{k}(x, B) .
$$

From the mean ergodic theorem and the individual ergodic theorem (Yosida (1980, p. 388)), for every $f \in L_{1}(v)$ there exists an $f^{*} \in L_{1}(v)$ such that

$$
G^{(n)} f \rightarrow f^{*} \quad \text { in } L_{1}(v) \text { as } n \rightarrow \infty \quad \text { and } \quad G f^{*}=f^{*} .
$$

For every $f \in L_{1}(v)$, the aforementioned function $f^{*}$ satisfies

$$
G^{(n)} f \rightarrow f^{*} \quad \text { v-a.e. as } n \rightarrow \infty \quad \text { and } \quad v\left(f^{*}\right)=v(f) .
$$

Consider a Borel right process $\left\{X_{t}\right\}_{t \in \mathbb{R}_{+}}$as defined in Section 2. Discrete-time Markov chains associated with this continuous-time process can be introduced. Denote by $\left\{\Phi_{k}\right\}_{k \in \mathbb{N}}$ the Markov chain associated with the resolvent kernel $R$ (defined in (2.2)). For a probability $F$ on $\mathbb{R}_{+}$, the Markov chain associated with the Markov kernel $K_{F}^{X}$ (defined in (2.1)) is denoted by $\left\{\Theta_{k}^{F}\right\}_{k \in \mathbb{N}}$. We have the following result. 
Lemma 3.1. If $A \in \mathscr{B}$ is such that $A=\mathcal{M}^{X}(A)$, then $\mathcal{M}^{X}(A)=\mathcal{M}^{\Phi}(A)$.

Proof. From Theorem 2.1(i) of Meyn and Tweedie (1993b), it is clear that $\mathcal{M}^{X}(A)=$ $\left\{x \in X: L^{\Phi}(x, A)=1\right\}$. From Proposition 9.1.1 of Meyn and Tweedie (1993a), and since $A=\mathcal{M}^{X}(A)=\left\{x \in X: L^{\Phi}(x, A)=1\right\}$, it follows that $\left\{x \in X: L^{\Phi}(x, A)=1\right\}=\mathcal{M}^{\Phi}(A)$, completing the proof.

In Theorem 10.19 of Blumenthal and Getoor (1968, p. 58), those authors showed that, under the hypothesis that the Markov process $\left\{X_{t}\right\}_{t \in \mathbb{R}_{+}}$is a Hunt process, the first hitting time of a Borel set can be approximated by a sequence of first hitting times of compact subsets. However, it can easily be shown that this result remains valid without this hypothesis.

Lemma 3.2. If $B$ is a Borel set then, for each probability measure $\mu$ on $(B, \mathcal{B})$, there exists an increasing sequence of compact subsets of $B$, denoted by $\left\{K_{n}\right\}$, such that $\tau_{K_{n}}^{X} \downarrow \tau_{B}^{X} \mathrm{P}_{\mu}$-almost surely.

Proof. The proof exactly follows that of Theorem 10.19 of Blumenthal and Getoor (1968, p. 58) except that the result about the first entry time given by Theorem A5.30 of Sharpe (1988, pp. 393-394) must be used instead of Corollary 10.17 of Blumenthal and Getoor (1968, p. 57).

We have the following result.

Lemma 3.3. Suppose that $E \in \mathcal{B}$ is an absorbing set for $R$. Then $\mathcal{M}^{X}\left(\mathcal{M}^{X}(E)\right)=\mathcal{M}^{X}(E)$ and $\mathcal{M}^{X}(E)$ is a maximal closed set for the continuous-time process $\left\{X_{t}\right\}_{t \in \mathbb{R}_{+}}$.

Proof. From Theorem 2.1(i) of Meyn and Tweedie (1993b), it is clear that

$$
\mathcal{M}^{X}(E)=\left\{x \in X: L^{\Phi}(x, E)=1\right\} .
$$

Let $D=\mathcal{M}^{X}(E)$. Since $E$ is absorbing for $R$, we have $E \subset D$. If $x \in D$ then, on the one hand, it follows that $L^{\Phi}(x, E)=1$; since obviously $L^{\Phi}(x, D)=1$, we thus have $x \in \mathcal{M}^{X}(D)$, showing that $D \subset \mathcal{M}^{X}(D)$. On the other hand, if $x \in \mathcal{M}^{X}(D)$ then, from the strong Markov property,

$$
L^{\Phi}(x, E)=\mathrm{P}_{x}\left(\tau_{E}^{\Phi}<\infty\right)=\mathrm{E}_{x}\left(L^{\Phi}\left(X_{\tau_{D}^{\Phi}}, E\right) \mathbf{1}_{\left\{\tau_{D}^{\Phi}<\infty\right\}}\right)=1
$$

and, thus, $x \in D$, showing that $\mathcal{M}^{X}(D) \subset D$. This gives the first part of the result.

From Lemma 3.1 it follows that $D=\mathcal{M}^{\Phi}(D)$, that is, $D$ is a maximal closed set for $R$. From Proposition 2.1 of Tuominen and Tweedie (1979), now using Lemma 3.2 instead of Theorem 10.19 of Blumenthal and Getoor (1968, p. 58), the result follows.

The following result is an adaptation of Theorem 2.1 of Tuominen and Tweedie (1979), in which is was assumed that $\left\{X_{t}\right\}_{t \in \mathbb{R}_{+}}$is a Hunt process. Here we relax this hypothesis by using Lemma 3.2 (note that here the process is assumed to be Borel right).

Theorem 3.1. For any set $A \in \mathcal{B}$,

(i) A is transient for $\left\{X_{t}\right\}_{t \in \mathbb{R}_{+}}$if and only if $A$ is transient for $\left\{\Phi_{k}\right\}_{k \in \mathbb{N}}$, and

(ii) A is a maximal Harris set for $\left\{X_{t}\right\}_{t \in \mathbb{R}_{+}}$if and only if $A$ is a maximal Harris set for $\left\{\Phi_{k}\right\}_{k \in \mathbb{N}}$.

Proof. The proof of this theorem follows the proofs of Theorem 2.1 and Proposition 2.1 of Tuominen and Tweedie (1979). Note that the hypothesis that $\left\{X_{t}\right\}_{t \in \mathbb{R}_{+}}$is a Hunt process appears only in the proof of Proposition 2.1 there, where Tuominen and Tweedie used the 
theorem of approximation of the first hitting times derived by Blumenthal and Getoor (see Theorem 10.19 of Blumenthal and Getoor (1968, p. 58)). However, now using Lemma 3.2 instead of Theorem 10.19 of Blumenthal and Getoor (1968, p. 58), the result follows.

\section{Limiting kernel}

In this section we present some convergence results for the expected occupation measure for the continuous-time process $\left\{X_{t}\right\}_{t \in \mathbb{R}_{+}}$, following an approach closely related to the one presented in Yosida (1980, pp. 393-397) and Hernández-Lerma and Lasserre (1998). In particular, we show that the limit kernel associated with the continuous-time process can be written $\mu$-a.e. (where $\mu$ is an IPM for the continuous-time process) as a composition of the limit kernel associated with an embedded discrete-time Markov chain of the location of the process at time 1 with an expected value operator of the integral of the process up to time 1 . We also obtain the weak convergence $\mu$-a.e. of the expected occupation measure for the continuous-time process without any Feller hypothesis (see, e.g. Yosida (1980, p. 393)) on the process.

We assume that an IPM $\mu$ exists for the continuous-time process $\left\{X_{t}\right\}_{t \in \mathbb{R}_{+}}$(and, thus, from Lemma 1 of Azéma et al. (1967), for the discrete-time process $\left\{\Phi_{k}\right\}_{k \in \mathbb{N}}$ associated with the resolvent $R$ ). We define the operator $g: L_{1}(\mu) \rightarrow L_{1}(\mu)$ as follows. For $f \in L_{1}(\mu)$,

$$
g f(x):=\mathrm{E}_{x}\left(\int_{0}^{1} f\left(X_{s}\right) \mathrm{d} s\right)=\int_{0}^{1} P^{s} f(x) \mathrm{d} s .
$$

Since $\mu(|f|)<\infty$, it is easy to verify that $\mu(|g f|)<\infty$. We denote by $R^{(n)}$ the $n$-step expected occupation measure associated with the kernel $R$, as defined (for the kernel $G$ ) in (3.1). Also define the stochastic kernel $Q$ by $Q:=P^{1}$. Clearly, $\mu$ is also invariant for $Q$, $\sum_{k=0}^{n-1} Q^{k} g f(x)=\mathrm{E}_{x}\left(\int_{0}^{n} f\left(X_{s}\right) \mathrm{d} s\right)$ for any $f \in L_{1}(\mu)$, and $\mu Q g=\mu$. The occupation measure $P^{(t)}$ for the continuous-time process $\left\{X_{t}\right\}_{t \in \mathbb{R}_{+}}$is defined by

$$
P^{(t)}(x, B):=\frac{1}{t} \int_{0}^{t} P^{s}(x, B) \mathrm{d} s .
$$

Let $\hat{\Pi}^{\prime}: L_{1}(\mu) \rightarrow L_{1}(\mu)$ be the Markov operator associated with the stochastic kernel $Q$ and let $\hat{\Pi}: L_{1}(\mu) \rightarrow L_{1}(\mu)$ be the Markov operator associated with the stochastic kernel $R$, as in Hernández-Lerma and Lasserre (1998). Similarly, let $\varphi_{x}^{\prime}$ be the measure associated with the stochastic kernel $Q$ and let $\varphi_{x}$ be the measure associated with the stochastic kernel $R$, as in Lemma 3.3 of Hernández-Lerma and Lasserre (1998). We have the following results.

Proposition 4.1. Let $x \in X$ be such that $Q^{(n)} g f(x)$ converges for any $f \in L_{1}(\mu)$. Then $\lim _{n \rightarrow \infty} Q^{(n)} g f(x)=\lim _{t \rightarrow \infty} P^{(t)} f(x)$.

Proof. For any $f \in L_{1}(\mu)$, let $f^{+}=\max \{f, 0\} \in L_{1}(\mu)$. Denote by $\lfloor t\rfloor$ the integer part of the positive real number $t$. For any $t \geq 1$,

$$
\begin{aligned}
\frac{\lfloor t\rfloor}{t} \frac{1}{\lfloor t\rfloor} \int_{0}^{\lfloor t\rfloor} \mathrm{E}_{x}\left(f^{+}\left(X_{s}\right)\right) \mathrm{d} s & \leq \frac{1}{t} \int_{0}^{t} \mathrm{E}_{x}\left(f^{+}\left(X_{s}\right)\right) \mathrm{d} s \\
& \leq \frac{\lfloor t\rfloor+1}{t} \frac{1}{\lfloor t\rfloor+1} \int_{0}^{\lfloor t\rfloor+1} \mathrm{E}_{x}\left(f^{+}\left(X_{s}\right)\right) \mathrm{d} s
\end{aligned}
$$

or, in other words, from (4.1),

$$
\frac{\lfloor t\rfloor}{t} Q^{(\lfloor t\rfloor)} g f^{+}(x) \leq P^{(t)} f^{+}(x) \leq \frac{\lfloor t\rfloor+1}{t} Q^{(\lfloor t\rfloor+1)} g f^{+}(x) .
$$


Taking the limit as $t \rightarrow \infty$, we obtain $\lim _{n \rightarrow \infty} Q^{(n)} g f^{+}(x)=\lim _{t \rightarrow \infty} P^{(t)} f^{+}(x)$. Similarly, in the same limit we have $\lim _{n \rightarrow \infty} Q^{(n)} g f^{-}(x)=\lim _{t \rightarrow \infty} P^{(t)} f^{-}(x)$, where $f^{-}=$ $-\min \{f, 0\} \in L_{1}(\mu)$. Therefore, $\lim _{n \rightarrow \infty} Q^{(n)} g f(x)=\lim _{t \rightarrow \infty} P^{(t)} f(x)$, which is the desired result.

Proposition 4.2. Let $f \in L_{1}(\mu)$ be a bounded function and let $x \in X$ be such that $P^{(t)} f(x)$ converges. Then, for every $n=1,2, \ldots, \lim _{t \rightarrow \infty} P^{(t)} f(x)=\lim _{t \rightarrow \infty} P^{(t)} R^{(n)} f(x)$.

Proof. From the Markov property we have $P^{t} R f(x)=\mathrm{e}^{t} \mathrm{E}_{x}\left(\int_{t}^{\infty} \mathrm{e}^{-s} f\left(X_{s}\right) \mathrm{d} s\right)$, and by integration by parts we have

$$
\int_{0}^{\tau} P^{t} f(x) \mathrm{d} t=\int_{0}^{\tau} P^{t} R f(x) \mathrm{d} t-\mathrm{e}^{\tau}\left(\int_{\tau}^{\infty} \mathrm{e}^{-s} P^{s} f(x) \mathrm{d} s\right)+R f(x) .
$$

Dividing this by $\tau$ and taking the limit as $\tau \rightarrow \infty$, from the fact that $f$ is bounded we obtain $\lim _{\tau \rightarrow \infty} P^{(\tau)} f(x)=\lim _{\tau \rightarrow \infty} P^{(\tau)} R f(x)$. By iterating this equation and adding the results we obtain

$$
n \lim _{\tau \rightarrow \infty} P^{(\tau)} f(x)=\lim _{\tau \rightarrow \infty} P^{(\tau)}\left(\sum_{k=1}^{n} R^{k}\right) f(x),
$$

from which the desired result follows.

Proposition 4.3. For any $f \in L_{1}(\mu)$ and $t \geq 0, P^{t} \hat{\Pi} f=\hat{\Pi} f$.

Proof. From Lemma 4.3 of Down et al. (1995), we have $\mathcal{A} R \hat{\Pi} f(x)=(R-I) \hat{\Pi} f(x)$ (see Definition 2.5), where $I$ represents the identity operator. However, since $(R-I) \hat{\Pi} f(x)=0$ (see Lemma 3.2 of Hernández-Lerma and Lasserre (1998)), it follows that $\mathcal{A} R \hat{\Pi} f(x)=\mathcal{A} \hat{\Pi} f(x)=$ 0 and, from (2.3), that $P^{t} \hat{\Pi} f(x)-f(x)=\int_{0}^{t} \mathrm{E}_{x}\left(\mathcal{A} \hat{\Pi} f\left(X_{s}\right)\right) \mathrm{d} s=0$, from which the desired result follows.

Proposition 4.4. For $\mu$-almost every $x$, we have $\varphi_{x}(B)=\varphi_{x}^{\prime} g(B)$ for all $B \in \mathcal{B}$.

Proof. Let $C_{0}(X)$ be the space of continuous functions on $X$ vanishing at infinity (recall that any $f \in C_{0}(X)$ is a bounded function). Let $X_{1} \in \mathscr{B}$ be such that Lemma 3.3(a)-(e) of Hernández-Lerma and Lasserre (1998) and (3.3) are satisfied for the kernels $Q$ and $R$. Then $\mu\left(X_{1}\right)=1$ and from Propositions 4.1 and 4.2 we have, for every $x \in X_{1}$ and $f \in C_{0}(X)$,

$$
\hat{\Pi}^{\prime} g f(x)=\lim _{t \rightarrow \infty} P^{(t)} f(x)=\lim _{t \rightarrow \infty} P^{(t)} R^{(n)} f(x)=\hat{\Pi}^{\prime} g R^{(n)} f(x)
$$

that is, for every $n=1,2, \ldots$ and $x \in X_{1}, \hat{\Pi}^{\prime} g f(x)=\hat{\Pi}^{\prime} g R^{(n)} f(x)$. Moreover, from (3.2), $R^{(n)} f \rightarrow \hat{\Pi} f$ in $L_{1}(\mu)$ as $n \rightarrow \infty$.

Let us now show that $g R^{(n)} f \rightarrow g \hat{\Pi} f$ in $L_{1}(\mu)$. Indeed,

$$
\begin{aligned}
\int_{X}\left|g R^{(n)} f(y)-g \hat{\Pi} f(y)\right| \mu(\mathrm{d} y) & \leq \int_{X} \int_{0}^{1} P^{s}\left(\left|R^{(n)} f-\hat{\Pi} f\right|\right)(y) \mathrm{d} s \mu(\mathrm{d} y) \\
& =\int_{X}\left(\left|R^{(n)} f-\hat{\Pi} f\right|\right)(y) \mu(\mathrm{d} y),
\end{aligned}
$$

yielding the desired convergence. According to Lemma 3.2 of Hernández-Lerma and Lasserre (1998), we have $\hat{\Pi}^{\prime} g R^{(n)} f \rightarrow \hat{\Pi}^{\prime} g \hat{\Pi} f$ in $L_{1}(\mu)$ as $n \rightarrow \infty$. However, notice that

$$
\hat{\Pi}^{\prime} g \hat{\Pi} f(y)=\lim _{\tau \rightarrow \infty} P^{(\tau)} \hat{\Pi} f(y)=\hat{\Pi} f(y),
$$


by Proposition 4.3. This means that $\hat{\Pi}^{\prime} g R^{(n)} f \rightarrow \hat{\Pi} f$ in $L_{1}(\mu)$ as $n \rightarrow \infty$. As seen above, we have $\hat{\Pi}^{\prime} g f(x)=\hat{\Pi}^{\prime} g R^{(n)} f(x)$ for $x \in X_{1}$, and $\mu\left(X_{1}\right)=1$, so

$$
0=\lim _{n \rightarrow \infty} \int_{X}\left|\hat{\Pi}^{\prime} g R^{(n)} f(x)-\hat{\Pi} f(x)\right| \mu(\mathrm{d} x)=\int_{X_{1}}\left|\hat{\Pi}^{\prime} g f(x)-\hat{\Pi} f(x)\right| \mu(\mathrm{d} x) .
$$

Therefore, from (4.2) there exists a set $N(f) \subseteq X_{1}, N(f) \in \mathcal{B}$, such that $\mu(N(f))=1$ and, for every $x \in N(f), \hat{\Pi}^{\prime} g f(x)=\hat{\Pi} f(x)$. Moreover, from Equation (3.12) of Hernández-Lerma and Lasserre (1998) and the definition of $X_{1}$, we have $\hat{\Pi}^{\prime} g f(x)=\varphi_{x}^{\prime} g f$ and $\hat{\Pi} f(x)=\varphi_{x} f$, where $\varphi_{x}^{\prime}$ and $\varphi_{x}$ are IPMs for $Q$ and $R$, respectively. Now let $\left\{g_{\ell}\right\}$ be a countable, dense set in $C_{0}(X)$ and set $N=\bigcap_{\ell} N\left(g_{\ell}\right)$. The denseness of $\left\{g_{\ell}\right\}$ means that $\varphi_{x}^{\prime} g g=\varphi_{x} g$ for every $g \in C_{0}(X)$ and $x \in N$, which implies the desired result.

We now prove the main theorem of this section, which traces a parallel with Theorem 3.1 of Hernández-Lerma and Lasserre (1998).

Theorem 4.1. Suppose that there exists an IPM $\mu$ for the continuous-time process $\left\{X_{t}\right\}_{t \in \mathbb{R}_{+}}$. Then there are stochastic kernels $\Pi(x, B)$ and $\bar{\Pi}(x, B)$ such that the following statements hold $\mu$-a.e. in $X$.

(a) $\Pi=\Pi P^{t}$ for all $t \geq 0, \bar{\Pi}=\bar{\Pi} Q$, and $\Pi=\bar{\Pi} g$.

(b) $\lim _{t \rightarrow \infty} P^{(t)} f=\Pi f$ for all $f \in L_{1}(\mu)$, and $P^{(t)}(x, \cdot)$ converges weakly to $\Pi(x, \cdot)$.

(c) $P^{t} \Pi=\Pi$ for each $t \geq 0$, ПП $=\Pi$, and $\mu$ is an IPM for $\Pi$.

Proof. As in Hernández-Lerma and Lasserre (1998), let $\Pi(x, \cdot)=\varphi_{x}(\cdot)$ if $x \in \operatorname{supp}(\mu)$ and let $\Pi(x, \cdot)=\mu^{0}$ otherwise, where $\operatorname{supp}(\mu)$ is the support of the measure $\mu$ and $\mu^{0}$ is any measure equivalent to $\mu$. Similarly, let $\bar{\Pi}(x, \cdot)=\varphi_{x}^{\prime}(\cdot)$ if $x \in \operatorname{supp}(\mu)$ and $\bar{\Pi}(x, \cdot)=\mu^{0}$ otherwise. Let us prove (a) first. As proved in Theorem 3.1 (a) of Hernández-Lerma and Lasserre (1998), $\Pi$ is an IPM for $R \mu$-a.e. in $X$ and, according to Lemma 1 of Azéma et al. (1967), $\Pi$ is an IPM for $P^{t} \mu$-a.e. in $X$. From Theorem 3.1(a) of Hernández-Lerma and Lasserre (1998) again, $\bar{\Pi}$ is an IPM for $Q \mu$-a.e. in $X$. The proof of (a) then follows from Proposition 4.4, due to the facts that $\Pi(x, \cdot)=\varphi_{x}(\cdot)$ and $\bar{\Pi}(x, \cdot)=\varphi_{x}^{\prime}(\cdot)$ for $x \in \operatorname{supp}(\mu)$.

Let us now prove (b). We have $g f \in L_{1}(\mu)$ for any $f \in L_{1}(\mu)$, and, from the definition of the set $N$ in Proposition 4.4 and the stochastic kernels $\Pi$ and $\bar{\Pi}$, for any $x \in N \cap \operatorname{supp}(\mu)$ we have $Q^{(n)} g f(x) \rightarrow \varphi_{x}^{\prime} g f=\varphi_{x} f=\Pi f(x)$. From Proposition 4.1, $\lim _{n \rightarrow \infty} Q^{(n)} g f(x)=$ $\lim _{t \rightarrow \infty} P^{(t)} f(x)$. Since $C_{\mathrm{b}}(X) \subset L_{1}(\mu)$ (where $C_{\mathrm{b}}(X)$ represents the set of real-valued continuous, bounded functions on $X$ ), the second part of (b) follows from the first.

Finally let us show (c). With $B=N \cap \operatorname{supp}(\mu)$, we have $\mu(B)=1=\int_{X} P^{t}(z, B) \mu(\mathrm{d} z)$. Therefore, for each $t$, we can find a set $C(t) \in \mathscr{B}$ such that $\mu(C(t))=1$ and $P^{t}(z, B)=1$ for every $z \in C(t)$. Now notice that $\hat{\Pi}(y, \cdot)=\Pi(y, \cdot)$ for $y \in B$ and that, from Proposition 4.3,

$$
\Pi f(x)=\hat{\Pi} f(x)=P^{t} \hat{\Pi} f(x)=\int_{B} \hat{\Pi} f(y) P^{t}(x, \mathrm{~d} y)=\int_{B} \Pi f(y) P^{t}(x, \mathrm{~d} y)=P^{t} \Pi f(x)
$$

for any $f \in L_{1}(\mu)$ and $x \in C(t) \cap B$. The first part of (c) follows. The remaining results follow from Theorem 3.1 of Hernández-Lerma and Lasserre (1998). 


\section{Yosida decomposition}

In this section we present the so-called Yosida decomposition for the continuous-time process $\left\{X_{t}\right\}_{t \in \mathbb{R}_{+}}$, following an approach closely related to the one presented in Yosida (1980, pp. 393397) and Hernández-Lerma and Lasserre (1998). In what follows we denote by $\delta^{X}$ the closed sets for $\left\{X_{t}\right\}_{t \in \mathbb{R}_{+}}$and, similarly, by $\delta^{\Phi}$ the closed sets (equivalent in this case to the absorbing sets) for the discrete-time process $\left\{\Phi_{k}\right\}_{k \in \mathbb{N}}$.

Remark 5.1. Note that $8^{X} \subset 8^{\Phi}$. Indeed, suppose that $E \in 8^{X}$, whence $P^{t}(x, E)=1$ for all $x \in E$ and all $t \in \mathbb{R}_{+}$. Then clearly $R(x, E)=1$ for all $x \in E$.

We now recall the following definition.

Definition 5.1. A measure $v$ on $\mathscr{B}$ is said to be ergodic if it is an IPM such that, for every closed set $B \in \mathscr{B}, v(B)=1$ or $v(B)=0$. We denote by $u^{X}$ the set of ergodic measures for $\left\{X_{t}\right\}_{t \in \mathbb{R}_{+}}$and by $\mathcal{U}^{\Phi}$ the set of ergodic measures for $\left\{\Phi_{k}\right\}_{k \in \mathbb{N}}$.

We have the following results.

Lemma 5.1. Assume that $v$ is an invariant measure for the Markov chain $\left\{\Phi_{k}\right\}_{k \in \mathbb{N}}$. Then $v(E)=v\left(\mathcal{M}^{X}(E)\right)$ for all $E \in \mathcal{s}^{\Phi}$.

Proof. For $E \in \mathcal{S}^{\Phi}$, we have $L^{\Phi}(x, E)=1$ and $E \subset \mathcal{M}^{X}(E)$. Define

$$
U_{E}(x, B):=\sum_{n=1}^{\infty}\left[\left(R \mathbf{1}_{E^{\mathrm{c}}}\right)^{n-1} R\right](x, B)
$$

for all $B \in \mathscr{B}$ and $x \in X$. Consequently, using Theorem 10.4.7 of Meyn and Tweedie (1993a), we find that the measure $v_{E}^{\circ}$ defined by $v_{E}^{\circ}(B)=\int_{E} U_{E}(y, B) v(\mathrm{~d} y)$ for all $B \in \mathscr{B}$ is invariant for the Markov chain $\left\{\Phi_{k}\right\}_{k \in \mathbb{N}}$, and, since $\mathcal{M}^{X}(E) \backslash E \subset\left\{x \in X: L^{\Phi}(x, E)>0\right\}$, we have $v\left(\mathcal{M}^{X}(E) \backslash E\right)=v_{E}^{\circ}\left(\mathcal{M}^{X}(E) \backslash E\right)$. However, since $E$ is absorbing, it follows that $U_{E}\left(y, \mathcal{M}^{X}(E) \backslash E\right)=0$ for all $y \in E$. Combining these results, we obtain $v\left(\mathcal{M}^{X}(E) \backslash E\right)=0$, from which the result follows.

Proposition 5.1. A measure $v$ is ergodic for the continuous-time process $\left\{X_{t}\right\}_{t \in \mathbb{R}_{+}}$if and only if $v$ is ergodic for the discrete-time process $\left\{\Phi_{k}\right\}_{k \in \mathbb{N}}$ associated with the resolvent $R$.

Proof. From Remark 5.1, the 'if' part is straightforward. To prove the converse implication, suppose that $v$ is ergodic for $\left\{X_{t}\right\}_{t \in \mathbb{R}_{+}}$but not for $\left\{\Phi_{k}\right\}_{k \in \mathbb{N}}$. In this case we can find an $E \in \varsigma^{\Phi}$ such that $0<v(E)<1$. Using Lemma 5.1, we find that $v(E)=v\left(\mathcal{M}^{X}(E)\right)$. However, from Lemma 3.3, $\mathcal{M}^{X}(E) \in \varsigma^{X}$. Consequently $0<v\left(\mathcal{M}^{X}(E)\right)<1$, implying that $v$ is not ergodic for $\left\{X_{t}\right\}_{t \in \mathbb{R}_{+}}$, which leads to a contradiction. Therefore, $v$ is ergodic for $\left\{\Phi_{k}\right\}_{k \in \mathbb{N}}$, giving the result.

The next result traces a parallel with Lemma 4.2 of Hernández-Lerma and Lasserre (1998).

Proposition 5.2. If the measure $v$ is ergodic for the continuous-time process $\left\{X_{t}\right\}_{t \in \mathbb{R}_{+}}$, then there exists a maximal closed set $\hat{E} \in \mathcal{s}^{X}$ such that $v(\hat{E})=1$. Moreover, with $\left\{\hat{X}_{t}\right\}_{t \in \mathbb{R}_{+}}$the restriction of $\left\{X_{t}\right\}_{t \in \mathbb{R}_{+}}$over $\hat{E}$, it follows that

(i) $v$ is the unique IPM of the continuous-time process $\left\{\hat{X}_{t}\right\}_{t \in \mathbb{R}_{+}}$, and

(ii) $\hat{E}$ is indecomposable. 
Proof. Let $\varphi_{x}$ be the measure associated with the resolvent $R$, as in Lemma 3.3 of Hernández-Lerma and Lasserre (1998), and let $\tilde{S}=\left\{x \in X: \varphi_{x}=v\right\} \cap\left\{x \in X: R^{(n)}(x, \cdot)\right.$ converges weakly to $\left.\varphi_{x}\right\}$. According to Theorem 3.1 and Lemma 4.2 of Hernández-Lerma and Lasserre (1998), we have $v(\tilde{S})=1$. Following the approach of Yosida (1980, pp. 393-397) (see also Hernández-Lerma and Lasserre (1998, p. 113)), we can find an absorbing set $\tilde{E} \in \varsigma^{\Phi}$ such that $\tilde{E} \subset \tilde{S}$ and $v(\tilde{E})=1$. Let $\hat{E}=\mathcal{M}(\tilde{E})=\left\{x \in X: L^{\Phi}(x, \tilde{E})=1\right\}$ (from Theorem 2.1(i) of Meyn and Tweedie (1993b)). From Lemma 3.3, $\hat{E} \in \varsigma^{X}$ and, clearly, $\tilde{E} \subset \hat{E}$, meaning that $v(\hat{E})=1$.

Let us show that $\hat{E} \subset \tilde{S}$. Consider an $x \in \hat{E}$ and any function $f \in C_{\mathrm{b}}(X)$ (recall that $C_{\mathrm{b}}(X)$ denotes the space of bounded continuous functions on $X$ ). We have

$$
\sum_{k=1}^{n} R^{k} f(x)=\mathrm{E}_{x}\left(\sum_{k=1}^{n} f\left(\Phi_{k}\right)\right)=\sum_{m=1}^{\infty} \mathrm{E}_{x}\left(\sum_{k=1}^{n} f\left(\Phi_{k}\right) \mathbf{1}_{\left\{\tau_{\tilde{E}}^{\Phi}=m\right\}}\right),
$$

since $\mathrm{P}_{x}\left(\tau_{\tilde{E}}^{\Phi}<\infty\right)=1$. However, from the Markov property it follows that, for $n>m$,

$$
\frac{1}{n} \mathrm{E}_{x}\left(\sum_{k=1}^{n} f\left(\Phi_{k}\right) \mathbf{1}_{\left\{\tau_{\tilde{E}}^{\Phi}=m\right\}}\right)=\frac{1}{n} \mathrm{E}_{x}\left(\sum_{k=1}^{m} f\left(\Phi_{k}\right) \mathbf{1}_{\left\{\tau_{\tilde{E}}^{\Phi}=m\right\}}\right)+\frac{1}{n} \mathrm{E}_{x}\left(\sum_{k=1}^{n-m} R^{k} f\left(\Phi_{m}\right) \mathbf{1}_{\left\{\tau_{\tilde{E}}^{\Phi}=m\right\}}\right) .
$$

Note that $\Phi_{\tau_{\tilde{E}}^{\Phi}} \in \tilde{E} \subset \tilde{S}$. By using the bounded convergence theorem, we obtain

$$
\lim _{n \rightarrow \infty} \frac{1}{n} \mathrm{E}_{x}\left(\sum_{k=1}^{n-m} R^{k} f\left(\Phi_{m}\right) \mathbf{1}_{\left\{\tau_{\tilde{E}}^{\Phi}=m\right\}}\right)=v(f) \mathrm{P}_{x}\left(\tau_{\tilde{E}}^{\Phi}=m\right) .
$$

Combining these results yields

$$
\lim _{n \rightarrow \infty} \frac{1}{n} \mathrm{E}_{x}\left(\sum_{k=1}^{n} f\left(\Phi_{k}\right) \mathbf{1}_{\left\{\tau_{\tilde{E}}^{\Phi}=m\right\}}\right)=v(f) \mathrm{P}_{x}\left(\tau_{\tilde{E}}^{\Phi}=m\right)
$$

and, again from the bounded convergence theorem, we obtain

$$
\lim _{n \rightarrow \infty} \frac{1}{n} \sum_{k=1}^{n} R^{k} f(x)=v(f) \sum_{m=1}^{\infty} \mathrm{P}_{x}\left(\tau_{\tilde{E}}^{\Phi}=m\right)=v(f) .
$$

Thus, $R^{(n)}(x, \cdot)$ converges weakly to $v$ and $x \in \tilde{S}$, implying that $\hat{E} \subset \tilde{S}$. From Lemma 3.3 of Hernández-Lerma and Lasserre (1998), for an arbitrary IPM $\mu$ for $P^{t}(x, \cdot)$ (and, thus, for $R$ ) such that $\mu(\hat{E})=1$, we have $\mu(B)=\int_{\hat{E}} \mu(\mathrm{d} x) \varphi_{x}(B)=v(B)$ for all $B \in \mathcal{B}$, proving item (i).

Now suppose that $\hat{E}$ can be decomposed as the union of two disjoint closed sets $A$ and $B$ with $v(A) v(B)>0$. Then, from Remark 5.1, $A$ and $B$ are disjoint absorbing sets for $R$, leading, according to the proof of Lemma 4.2(h) of Hernández-Lerma and Lasserre (1998), to a contradiction.

As in Hernández-Lerma and Lasserre (1998), let $\mu$ be an IPM for $P^{t}(x, \cdot)$ and define

$$
\Lambda\left(f, \varphi_{x}\right)=\int_{X}\left(\varphi_{x}(f)-\mu(f)\right)^{2} \mu(\mathrm{d} x),
$$

define $X_{1}$ as the set of points in $X$ such that Lemma 3.3(a)-(e) of Hernández-Lerma and Lasserre (1998) holds, and define $X_{2}=\left\{x \in X_{1}: \Lambda\left(f, \varphi_{x}\right)=0\right.$ for all $\left.f \in C_{0}(X)\right\}$. The following result presents the so-called Yosida decomposition for the continuous-time process $\left\{X_{t}\right\}_{t \in \mathbb{R}_{+}}$. 
Theorem 5.1. Let $\mu$ be an arbitrary IPM for the continuous-time process $\left\{X_{t}\right\}_{t \in \mathbb{R}_{+}}$, define $\varphi_{x}$ as in Lemma 3.3 of Hernández-Lerma and Lasserre (1998), and let $X_{2}$ be as defined above. Then, for every $x \in X_{2}$, there is a maximal closed set $\hat{E}_{x} \in 8^{X}$ such that $\varphi_{x}\left(\hat{E}_{x}\right)=1$. Moreover, with $\left\{\hat{X}_{t}\right\}_{t \in \mathbb{R}_{+}}$the restriction of $\left\{X_{t}\right\}_{t \in \mathbb{R}_{+}}$over $\hat{E}_{x}$, it follows that

(i) $\varphi_{x}$ is the unique IPM of the continuous-time process $\left\{\hat{X}_{t}\right\}_{t \in \mathbb{R}_{+}}$, and

(ii) $\hat{E}_{x}$ is indecomposable.

Proof. As defined in Hernández-Lerma and Lasserre (1998, pp. 114-115), $\varphi_{x}$ is an ergodic probability measure for $R$. From Proposition 5.1, $\varphi_{x}$ is an ergodic probability measure for the continuous-time process $\left\{X_{t}\right\}_{t \in \mathbb{R}_{+}}$, and the result follows from Proposition 5.2 with $v$ replaced by $\varphi_{x}$.

\section{Doeblin decomposition and Lyapunov criterion}

We start by recalling the definition of an everywhere-nontrivial $T$-continuous component for a stochastic kernel $K$, and that of $T$-processes (see Meyn and Tweedie (1993b)).

Definition 6.1. Let $K(\cdot, \cdot): X \times \mathscr{B} \rightarrow \mathbb{R}_{+}$be a stochastic kernel. A kernel $T(\cdot, \cdot): X \times \mathscr{B} \rightarrow$ $\mathbb{R}_{+}$is called a $T$-continuous component nontrivial everywhere on $X$ if

(i) $T(x, \cdot) \leq K(x, \cdot)$ for all $x \in X$,

(ii) $0<T(x, X) \leq 1$ for all $x \in X$, and

(iii) for each $A \in \mathcal{B}, T(\cdot, A)$ is a lower-semicontinuous function.

Definition 6.2. A continuous-time Markov process $\left\{X_{t}\right\}_{t \in \mathbb{R}_{+}}$will be called a $T$-process if there exists a probability $F$ on $\mathbb{R}_{+}^{*}$ such that the stochastic kernel $K_{F}^{X}$ admits an everywhere-nontrivial $T$-continuous component. A discrete-time Markov chain $\left\{\Psi_{n}\right\}_{n \in \mathbb{N}}$ will be called a $T$-process if there exists a probability $b$ on $\mathbb{N}^{*}$ such that the stochastic kernel $K_{b}^{\Psi}$ admits an everywherenontrivial $T$-continuous component.

The following definitions are related to the $T^{\prime}$-condition presented by Costa and Dufour (2005b), and generalize the concepts of $T$-continuous component and $T$-process (see the examples below).

Definition 6.3. ( $T^{\prime}$-component.) Let $K(\cdot, \cdot): X \times \mathscr{B} \rightarrow \mathbb{R}_{+}$be the stochastic kernel associated with the Markov chain $\left\{\Psi_{k}\right\}_{k \in \mathbb{N}}$. This kernel admits a $T^{\prime}$-component if there exist a probability $b$ on $\mathbb{N}^{*}$ and a kernel $T(\cdot, \cdot): X \times \mathscr{B} \rightarrow[0,1]$ such that

(i) $T(x, \cdot) \ll K_{b}^{\Psi}(x, \cdot)$ for all $x \in X$,

(ii) $0<T(x, X) \leq 1$ for all $x \in X$, and

(iii) there exist an $\mathcal{I} \subseteq \mathbb{N}_{*}$ and a countable sequence of sets $\left\{B_{n}\right\}_{n \in \mathcal{I}}$ in $\mathscr{B}$ such that, for every absorbing set $E$ of the Markov chain $\left\{\Psi_{k}\right\}_{k \in \mathbb{N}}$, there is an $\mathcal{I}(E) \subseteq \mathcal{I}$ such that

$$
\{x \in X: T(x, E)>0\}=\bigcup_{i \in \mathcal{X}(E)} B_{i} .
$$

Definition 6.4. ( $T^{\prime}$-process.) A continuous-time Markov process $\left\{X_{t}\right\}_{t \in \mathbb{R}_{+}}$where the associated transition semigroup is denoted by $\left\{P^{t}\right\}_{t \in \mathbb{R}_{+}}$will be called a $T^{\prime}$-process if there exists a 
distribution $F$ on $\mathbb{R}_{+}^{*}$ such that the stochastic kernel $K_{F}^{X}$ admits a $T^{\prime}$-component. A discretetime Markov chain $\left\{\Psi_{n}\right\}_{n \in \mathbb{N}}$ where the associated transition probability function is denoted by $G$ will be called a $T^{\prime}$-process if there exists a distribution $F$ on $\mathbb{R}_{+}^{*}$ such that the stochastic kernel $G$ admits a $T^{\prime}$-component.

Next, we present three examples of Markov processes that are $T^{\prime}$-processes. The first two examples show that standard classes of Markov process (such as irreducible processes or $T$-processes) are $T^{\prime}$-processes. In the last example it is shown that the class of processes satisfying the $T^{\prime}$-condition is wider than the class of irreducible processes and $T$-processes.

Example 6.1. If $\left\{X_{t}\right\}_{t \in \mathbb{R}_{+}}$is $\varphi$-irreducible then it is a $T^{\prime}$-process. Indeed, from the definition of $\varphi$-irreducibility, if for some $\varphi$ we have $\varphi(B)>0$, then $\mathrm{E}_{x}\left(\eta_{B}^{X}\right)>0$, and from Theorem 2.1(i) of Meyn and Tweedie (1993b) we have $L^{\Phi}(x, B)>0$, where $\left\{\Phi_{k}\right\}_{k \in \mathbb{N}}$ denotes the Markov chain associated with the resolvent. Consequently, it follows that $\left\{\Phi_{k}\right\}_{k \in \mathbb{N}}$ is $\varphi$-irreducible. As shown by Costa and Dufour (2005b), $\left\{\Phi_{k}\right\}_{k \in \mathbb{N}}$ is a $T^{\prime}$-chain, and from Definition $6.3\left\{X_{t}\right\}_{t \in \mathbb{R}_{+}}$ is a $T^{\prime}$-process.

Example 6.2. If $\left\{X_{t}\right\}_{t \in \mathbb{R}_{+}}$is a $T$-process then, for some distribution $F$, it is immediate that the stochastic kernel $K_{F}^{X}$ admits a $T^{\prime}$-component and, from Definition 6.3, that $\left\{X_{t}\right\}_{t \in \mathbb{R}_{+}}$is a $T^{\prime}$-process.

Example 6.3. Consider a scalar Markov jump linear system given by

$$
\dot{z}(t)=a_{\theta(t)} z(t), \quad z(0) \in \mathbb{R}, \quad \theta(t) \in N, a_{1}=-1, a_{2}=-2,
$$

where $N=\{1,2\}, \theta(t)$ is a continuous-time Markov process with jump rates $\lambda_{12}=1, \lambda_{21}=1$, and a dot denotes a time derivative. Note that this class of models has been the subject of extensive research over the last few years, and the associated literature is now fairly extensive. The interested reader may consult the following references (and the references cited therein): in the discrete-time context see Costa et al. (2004), and in the continuous-time context see Fragoso and Rocha (2005).

It is easy to see that $z(t)=\exp \left\{\int_{0}^{t} a_{\theta(s)} \mathrm{d} s\right\} z(0)$, and that $z(t)$ is a strictly decreasing function of $t$ for $z(0)>0$ and a strictly increasing function of $t$ for $z(0)<0$. We define the Markov process $\left\{X_{t}\right\}_{t \in \mathbb{R}_{+}}$, with $X_{t}=(z(t), \theta(t))$ taking values in $X=\mathbb{R} \times N$.

For any $i \in N$, let $x_{0}=(0, i), A_{0}=\{0\} \times N$, and $x_{n}=(1 / n, i)$. For any $T$-continuous component of $K_{F}^{X}$, we have $0=K_{F}^{X}\left(x_{n}, A_{0}\right) \geq T\left(x_{n}, A_{0}\right) \geq 0$ and, thus,

$$
0=\lim _{n \rightarrow \infty} T\left(x_{n}, A_{0}\right) \geq T\left(x_{0}, A_{0}\right) \geq 0,
$$

meaning that $T\left(x_{0}, A_{0}\right)=0$ (and, thus, $\left.T\left(x_{0},(\mathbb{R} \backslash\{0\}) \times N\right)>0\right)$, which is a contradiction since clearly $0=K_{F}^{X}\left(x_{0},(\mathbb{R} \backslash\{0\}) \times N\right) \geq T\left(x_{0},(\mathbb{R} \backslash\{0\}) \times N\right)$. Therefore, with the topology generated by the usual metric, we conclude that this process is not a $T$-process.

For $x=(z, i) \in \mathbb{R} \times N$ and $A$ any Borel set of $\mathbb{R}$, let

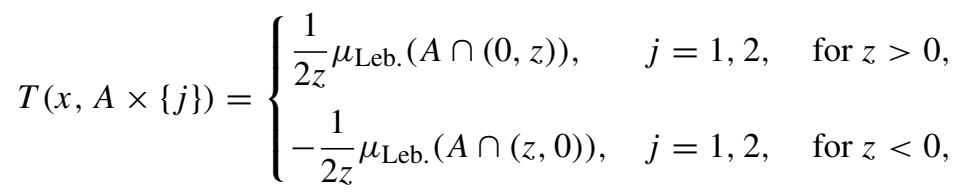

$$
\begin{aligned}
& T(x,\{0\} \times\{j\})=\frac{1}{2}, \quad j=1,2, \quad \text { for } z=0 .
\end{aligned}
$$


Also, let $F(t)$ be the exponential distribution with rate 1 , let $\left\{B_{n}\right\}_{n=2}^{\infty}$ be a countable basis of the open sets in $\mathbb{R}$, and let $B_{1}=\{0\}$. With these choices we see that $\left\{\Phi_{k}\right\}_{k \in \mathbb{N}}$ is a $T^{\prime}$-chain with $b_{1}=1$ and, thus, that $\left\{X_{t}\right\}_{t \in \mathbb{R}_{+}}$is a $T^{\prime}$-process.

The next result shows that a $T^{\prime}$-process admits a Doeblin decomposition.

Proposition 6.1. Assume that $\left\{X_{t}\right\}_{t \in \mathbb{R}_{+}}$is a Borel right process. It is then a $T^{\prime}$-process if and only if the Markov chain $\left\{\Phi_{k}\right\}_{k \in \mathbb{N}}$ is a $T^{\prime}$-chain. If $\left\{X_{t}\right\}_{t \in \mathbb{R}_{+}}$is a $T^{\prime}$-process, then it admits a Doeblin decomposition.

Proof. Let us first show that if $\left\{X_{t}\right\}_{t \in \mathbb{R}_{+}}$is a $T^{\prime}$-process then the Markov chain $\left\{\Phi_{k}\right\}_{k \in \mathbb{N}}$ is a $T^{\prime}$-chain. From Definition 6.4, if $\left\{X_{t}\right\}_{t \in \mathbb{R}_{+}}$is a $T^{\prime}$-process then there exists a distribution $F$ on $\mathbb{R}_{+}^{*}$ such that the stochastic kernel $K_{F}^{X}$ admits a $T^{\prime}$-component. According to Corollary 2.19 of Costa and Dufour (2005b), there exist a countably generated and separated $T_{1}$ topology consisting of all open sets in $\mathcal{B}$ and a probability $d$ on $\mathbb{N}^{*}$ such that $\sum_{k=1}^{\infty} d_{k}\left(K_{F}^{X}\right)^{k}$ admits a $T$-continuous component that is nontrivial everywhere on $X$ (or, in other words, $K_{H}^{X}$ admits a $T$-continuous component that is nontrivial everywhere on $X$, where $H=\sum_{k=1}^{\infty} d_{k} F^{* k}$ and $F^{* n}$ represents the $n$-fold convolution, since $\left.\sum_{k=1}^{\infty} d_{k}\left(K_{F}^{X}\right)^{k}=K_{H}^{X}\right)$. Now, according to Proposition 3.1 of Meyn and Tweedie (1993b), there exists a distribution $G$ on $\mathbb{R}_{+}^{*}$ that has a bounded density $g$ with respect to the Lebesgue measure and for which $K_{G}^{X}$ admits an everywherenontrivial continuous component with respect to the $T_{1}$ topology. We denote by $\left\{\tilde{\Phi}_{k}\right\}_{k \in \mathbb{N}}$ the Markov chain that is generated from the stochastic kernel $K_{G}^{X}$. Again using Corollary 2.19 of Costa and Dufour (2005b), it follows that $\left\{\tilde{\Phi}_{k}\right\}_{k \in \mathbb{N}}$ is a $T^{\prime}$-chain. Consequently, there exist a probability $b$ on $\mathbb{N}^{*}$ and a kernel $T(\cdot, \cdot)$ such that conditions (i), (ii), and (iii) of Definition 6.3 are satisfied.

Let us show that $T(x, \cdot) \ll R(x, \cdot)$ for all $x \in X$. Indeed, suppose that $A \in \mathcal{B}$ is such that $R(x, A)=\int_{0}^{\infty} P^{t}(x, A) \mathrm{e}^{-t} \mathrm{~d} t=0$. Then

$$
\sum_{k=1}^{\infty} b_{k}\left(K_{G}^{X}\right)^{k}(x, A)=\int_{0}^{\infty} P^{t}(x, A) \sum_{k=1}^{\infty} b_{k} g^{* k}(t) \mathrm{d} t=0,
$$

implying that $T(x, A)=0$. Moreover, if $E$ is an absorbing set of $\left\{\Phi_{k}\right\}_{k \in \mathbb{N}}$ then $E$ is an absorbing set of $\left\{\tilde{\Phi}_{k}\right\}_{k \in \mathbb{N}}$. Indeed, suppose that $E$ is an absorbing set of $\left\{\Phi_{k}\right\}_{k \in \mathbb{N}}$. Then, for every $x \in E$, we have $R(x, E)=1$, meaning that $R\left(x, E^{\mathrm{c}}\right)=\int_{0}^{\infty} P^{t}\left(x, E^{\mathrm{c}}\right) \mathrm{e}^{-t} \mathrm{~d} t=0$ and, thus, that $K_{G}^{X}\left(x, E^{\mathrm{c}}\right)=\int_{0}^{\infty} P^{t}\left(x, E^{\mathrm{c}}\right) g(t) \mathrm{d} t=0$, whence $K_{G}^{X}(x, E)=1$ for every $x \in E$. Now, it is easy to see that the resolvent $R$ satisfies Definition 6.3 for the $T^{\prime}$-component $T$. It thus follows that $\left\{\Phi_{k}\right\}_{k \in \mathbb{N}}$ is a $T^{\prime}$-chain.

Conversely, if $\left\{\Phi_{k}\right\}_{k \in \mathbb{N}}$ is a $T^{\prime}$-chain then it is easy to see, by taking $\mathrm{d} F(t)=\mathrm{e}^{-t} \mathrm{~d} t$, that $K_{F}=R$ admits a $T^{\prime}$-component and, thus, that $\left\{X_{t}\right\}_{t \in \mathbb{R}_{+}}$is a $T^{\prime}$-process, from which the first part of the result follows.

The second part of the result is a straightforward combination of the first part, Theorem 3.1 (here) and Theorem 2.18 of Costa and Dufour (2005b).

The next result shows that the Lyapunov criterion is necessary and sufficient for the existence of an IPM for a nonexplosive Borel right $T^{\prime}$-process. For the Borel right process $\left\{X_{t}\right\}_{t \in \mathbb{R}_{+}}$ (which will be assumed nonexplosive in the sequel), the extended generator is as defined in Definition 2.5.

Theorem 6.1. Assume that $\left\{X_{t}\right\}_{t \in \mathbb{R}_{+}}$is a nonexplosive Borel right process satisfying the $T^{\prime}$ condition. Then $\left\{X_{t}\right\}_{t \in \mathbb{R}_{+}}$admits an IPM if and only if there exist a petite set $C$ and a closed 
set $H$ for $\left\{X_{t}\right\}_{t \in \mathbb{R}_{+}}$, a measurable function $W: X \rightarrow \mathbb{R}_{+}$in the domain of $\mathcal{A}$, and a constant $d \in \mathbb{R}_{+}$such that

$$
\mathcal{A} W(x) \leq-1+d \mathbf{1}_{C}(x) \text { for all } x \in H .
$$

Proof. If there exists an IPM for $\left\{X_{t}\right\}_{t \in \mathbb{R}_{+}}$then, according to Lemma 1 of Azéma et al. (1967), there exists an invariant probability for the Markov chain $\left\{\Phi_{k}\right\}_{k \in \mathbb{N}}$ associated with the resolvent $R$. Using Proposition 6.1, we find that $\left\{\Phi_{k}\right\}_{k \in \mathbb{N}}$ is a $T^{\prime}$-chain. Consequently, following the proof of Proposition 5.1 of Costa and Dufour (2005b), there exists a maximal Harris set $H$ for $\left\{\Phi_{k}\right\}_{k \in \mathbb{N}}$ such that

$$
R V(x) \leq V(x)-1+d \mathbf{1}_{C}(x) \text { for all } x \in H,
$$

where $d \in \mathbb{R}_{+}, C$ is a petite set for $R$, and $V: X \rightarrow \overline{\mathbb{R}}_{+}=\mathbb{R}_{+} \cup\{\infty\}$ is a measurable function with $V(x)<\infty$ for all $x \in H$. Denote by $\hat{V}$ the restriction of $V$ to $H$. Since $H$ is a maximal Harris set for $\left\{\Phi_{k}\right\}_{k \in \mathbb{N}}$, it is also a maximal Harris set for $\left\{X_{t}\right\}_{t \in \mathbb{R}_{+}}$, by Theorem 3.1. Consider the restrictions $\left\{\hat{X}_{t}\right\}_{t \in \mathbb{R}_{+}}$and $\left\{\hat{\Phi}_{k}\right\}_{k \in \mathbb{N}}$ of the process $\left\{X_{t}\right\}_{t \in \mathbb{R}_{+}}$and, respectively, the Markov chain $\left\{\Phi_{k}\right\}_{k \in \mathbb{N}}$ to $H$. Moreover, denote by $\hat{\mathcal{A}}$ the generator of the process $\left\{\hat{X}_{t}\right\}_{t \in \mathbb{R}_{+}}$. Clearly $\left\{\hat{X}_{t}\right\}_{t \in \mathbb{R}_{+}}$is a nonexplosive Borel right process, and we denote by $\hat{R}$ its associated resolvent. It is easy to show that $R V(x)=\hat{R} \hat{V}(x)$ for all $x \in H$. From (6.2), it follows that $\hat{V}$ is in the domain of $\hat{R}$; from Lemma 4.3 of Down et al. (1995) we thus find that

$$
\hat{\mathcal{A}} \hat{R} \hat{V}(x)=(\hat{R}-I) \hat{V}(x) \quad \text { for all } x \in H .
$$

Define the function $W: X \rightarrow \mathbb{R}_{+}$by

$$
W(x):= \begin{cases}\hat{R} \hat{V}(x) & \text { for } x \in H, \\ 0 & \text { for } x \in H^{\mathrm{c}}\end{cases}
$$

Clearly $W$ is a measurable function. Since $H$ is a closed set for $\left\{X_{t}\right\}_{t \in \mathbb{R}_{+}}$, we have

$$
\mathrm{E}_{x}\left(W\left(X_{t}\right)\right)=W(x)+\mathrm{E}_{x}\left(\int_{0}^{t}(R-I) V\left(X_{s}\right) \mathrm{d} s\right) \text { for all } x \in H,
$$

implying that $\mathcal{A} W(x)=(R-I) V(x)$ for all $x \in H$. Moreover, $\mathcal{A} W(x)=0$ for all $x \in H^{\mathrm{c}}$. Therefore, $W$ is in the domain of $\mathcal{A}$. Finally, using (6.2), we find that

$$
\mathcal{A} W(x) \leq-1+d \mathbf{1}_{C}(x) \text { for all } x \in H,
$$

where $C$ is a petite set for $\left\{X_{t}\right\}_{t \in \mathbb{R}_{+}}$(since it is petite for $R$ ), from which the 'if' part of the result follows.

The converse result is Theorem 4.1 of Costa and Dufour (2005a).

Before presenting our final result, we recall the following definition.

Definition 6.5. An IPM $\mu$ is said to be singular with respect to $P^{t}(x, \cdot)$ if, for each $x \in X$, there exists a set $E_{x}$ such that $\mu\left(E_{x}\right)=1$ and $\int_{0}^{\infty} P^{t}\left(x, E_{x}\right) \mathrm{d} t=0$. Otherwise $\mu$ is said to be nonsingular with respect to $P^{t}(x, \cdot)$. Similarly, an IPM $\mu$ is said to be singular with respect to $R(x, \cdot)$ if, for each $x \in X$, there exists a set $E_{x}$ such that $\mu\left(E_{x}\right)=1$ and $\sum_{k=1}^{\infty} R^{k}\left(x, E_{x}\right)=0$. Otherwise $\mu$ is said to be nonsingular with respect to $R(x, \cdot)$.

Theorem 6.2. If $\left\{X_{t}\right\}_{t \in \mathbb{R}_{+}}$is a $T^{\prime}$-process and an IPM $\mu$ exists for it, then every IPM is nonsingular with respect to $P^{t}(x, \cdot)$ and $U^{X}$ is countable. 
Proof. From Proposition 6.1 and the hypothesis that $\left\{X_{t}\right\}_{t \in \mathbb{R}_{+}}$is a $T^{\prime}$-process, it follows that $\left\{\Phi_{k}\right\}_{k \in \mathbb{N}}$ is a $T^{\prime}$-chain. From Theorem 2.21 of Costa and Dufour (2005b), $\mathcal{U}^{\Phi}$ is countable and every IPM for $\left\{\Phi_{k}\right\}_{k \in \mathbb{N}}$ is nonsingular with respect to $R(x, \cdot)$. From Proposition 5.1, $u^{X}=U^{\Phi}$, from which the first part of the result follows. The second part follows from the identity $\int_{0}^{\infty} P^{t}\left(x, E_{x}\right) \mathrm{d} t=\sum_{k=1}^{\infty} R^{k}\left(x, E_{x}\right)$ and Lemma 1 of Azéma et al. (1967).

\section{Acknowledgements}

This work was supported by CAPES/COFECUB grant 425/03. The first author received financial support from CNPq (the Brazilian National Council for Scientific and Technological Development), grants 472920/03-0 and 304866/03-2; FAPESP (the State of São Paulo Research Foundation), grant 03/06736-7; PRONEX, grant 015/98; and IM-AGIMB.

\section{References}

AzÉma, J., Kaplan-Duflo, M. and Revuz, D. (1967). Mesure invariante sur les classes récurrentes des processus de Markov. Z. Wahrscheinlichkeitsth. 8, 157-181.

Blumenthal, R. M. and Getoor, R. K. (1968). Markov Processes and Potential Theory. Academic Press, New York.

Costa, O. L. V. AND Dufour, F. (2005a). A sufficient condition for the existence of an invariant probability measure for Markov processes. J. Appl. Prob. 42, 873-878.

Costa, O. L. V. And Dufour, F. (2005b). On the ergodic decomposition for a class of Markov chains. Stoch. Process. Appl. 115, 401-415.

Costa, O. L. V., Fragoso, M. D. and Marques, R. P. (2004). Discrete-Time Markov Jump Linear Systems. Springer, London.

Down, D., Meyn, S. P. And Tweedie, R. L. (1995). Exponential and uniform ergodicity of Markov processes. Ann. Prob. 23, 1671-1691.

Fragoso, M. D. and Rocha, N. C. S. (2005). Stationary filter for continuous-time Markovian jump linear systems. SIAM J. Control Optimization 44, 801-815.

Hernández-Lerma, O. ANd Lasserre, J. B. (1998). Ergodic theorems and ergodic decomposition for Markov chains. Acta Appl. Math. 54, 99-119.

Meyn, S. P. And Tweedie, R. L. (1992). Stability of Markovian processes. I. Criteria for discrete-time chains. Adv. Appl. Prob. 24, 542-574.

Meyn, S. P. And Tweedie, R. L. (1993a). Markov Chains and Stochastic Stability. Springer, Berlin.

Meyn, S. P. And Tweedie, R. L. (1993b). Stability of Markovian processes. II. Continuous-time processes and sampled chains. Adv. Appl. Prob. 25, 487-517.

Meyn, S. P. AND TwEedIE, R. L. (1993c). Stability of Markovian processes. III. Foster-Lyapunov criteria for continuoustime processes. Adv. Appl. Prob. 25, 518-548.

Meyn, S. P. And Tweedie, R. L. (1993d). The Doeblin decomposition. In Doeblin and Modern Probability (Contemp. Math. 149), American Mathematical Society, Providence, RI, pp. 211-225.

Sharpe, M. (1988). General Theory of Markov Processes. Academic Press, London.

Tuominen, P. And Tweedie, R. L. (1979). The recurrence structure of general Markov processes. Proc. London Math. Soc. 39, 554-576.

TweEdie, R. L. (1979). Topological aspects of Doeblin decompositions for Markov chains. Z. Wahrscheinlichkeitsth. 46, 299-305.

Yosida, K. (1980). Functional Analysis, 6th edn. Springer, New York. 